\title{
Demographic study of hemorrhoid with analysis of risk factors
}

\author{
Malviya V.K. ${ }^{1}$, Diwan S. ${ }^{2}$, Sainia T.K. ${ }^{3}$, Apte A. ${ }^{4}$ \\ ${ }^{1}$ Dr. Vikas Kumar Malviya, Assistant Professor, ${ }^{2}$ Dr. Shivam Diwan, Post Graduate Student, First year, ${ }^{3}$ Dr. Tarun \\ Kumar Sainia, Associate Professor, ${ }^{4}$ Dr. Ashwin Apte, Professor, all authors are affiliated with Department of General \\ Surgery, L.N. Medical College Bhopal, India.
}

Corresponding Author: Dr. Shivam Diwan, General Surgery, RSO $1^{\text {st }}$ Year, Post Graduate Student First year Department of General Surgery, L.N. Medical College Bhopal, India. E-mail: shivamdiwan7491@gmail.com

\begin{abstract}
Background: Hemorrhoids are the most prevalent benign anorectal disorder diagnosed in clinical practice. Risk factors commonly associated with hemorrhoidal disease include low fiber diet, chronic constipation \& diarrhea, straining during defecation, pregnancy, sedentary lifestyle, obesity etc. Treatment of symptomatic first-degree, second degree and early third-degree hemorrhoids includes banding and sclerotherapy. Patients with fourth-degree or large third-degree hemorrhoids should be referred for hemorrhoidectomy surgery. Methods: This retrospective study was conducted in the department of surgery in J.K. Hospital Bhopal. 430 adult patients with diagnosis of hemorrhoids, admitted in surgery ward were included in this study. All the relevant details were obtained from medical record department with all demographic details. Results: Most of the patients (46\%) belongs to the younger age (20-40 years). $69 \%$ were male patients and $31 \%$ were female patients. Patients with higher socioeconomic status were most commonly affected group (37.2\%) with hemorrhoids. Commonest symptoms was Bleeding per rectum. In the present study, risk factors for hemorrhoids were low fiber diet, mixed diet, poor hydration, chronic constipation or diarrhea, straining during the defecation, low physical activity and obesity. Sclerotherapy (11.1\%) and rubber band ligation (4.1\%) were non operative treatment. Operative procedures performed in the present study were open hemorrhoidectomy $(81.4 \%)$ and stapled hemorrhoidopexy (3.2\%). Conclusion: This demographic study of hemorrhoidal disease in this region can guide us to better understand the trends of this disease which is most commonly encountered in our society.This useful information may aid in the assessment and definitive care of these patients with haemorrhoids.
\end{abstract}

Keywords: Haemorrhoids; Demographic study; Risk factor

\section{Introduction}

Hemorrhoids or piles are bundles of vascular structure that contain enlarged blood vessels present within elastic fibers, smooth muscles and connective tissue of anus and anal canal. Usually these hemorrhoids work as venous cushions in normal individual and are the common cause of anal pathology [1].

These venous cushions help in control of stool defecation, but whenever there is a swelling or inflammation in these cushions, these become a pathologically a disease known as hemorrhoids. Hemorrhoid present as swelling, itching, pain during defecations and bleeding per rectum. Hemorrhoids are the most common benign anorectal disorder diagnosed in clinical practice and constitute about $50 \%$ of colorectal investigations [2]. Hemorrhoids are so much

Manuscript Received: $10^{\text {th }}$ January 2019

Reviewed: $20^{\text {th }}$ January 2019

Author Corrected: $27^{\text {th }}$ January 2019

Accepted for Publication: $30^{\text {th }}$ January 2019 common in India that every perianal disease is termed as hemorrhoid by most of the patients. Although hemorrhoids are so common, only few patients seek medical treatment due to embarrassment. Hemorrhoids are more common in the adult population. Men are more frequently affected in comparison to women [3].

Hemorrhoids are categorized as internal and external depending on location. Pectinate or dentate line that divides upper two thirds and lower one third of the anus is the demarcation line between external and internal hemorrhoids. External hemorrhoids are covered by skin and located below this line. Internal hemorrhoids are covered by mucosa and are located above the pectinate line. Internal hemorrhoids are true hemorrhoid with various degrees depending upon extent of protrusion out of anal canal and are located at 3,7 and 11 o' clock position. 


\section{Original Research Article}

Internal hemorrhoids are generally classified into first, second, third and fourth degree according to the classification published by Banov et al [4]. First degree hemorrhoids don't protrude out of the anal canal and may present as bleeding per rectum and discomfort.

Second degree hemorrhoids protrude with defecation and retract on their own and the patient may present as obvious swelling that disappears spontaneously and rapidly after defecation.

Third-degree hemorrhoids protrude and usually require manual replacement. Fourth degree hemorrhoids protrude all the time and cannot be reduced manually and present as persistent prolapsed pile.

Risk factors commonly associated with hemorrhoidal disease include low fiber diet, chronic constipation, chronic diarrhea, chronic straining during defecation, pregnancy, sedentary lifestyle, habit of postponing the bowel movements, obesity and spinal cord injuries etc.

Prevention is better than cure in cases of the hemorrhoids. Several treatment options are available for patients with hemorrhoid, who do not respond to conservative medical management [5-8]. Treatment guidelines are available from the American College of Gastroenterology (ACG) [9].

Treatment guidelines of American College of Gastroenterology (ACG) recommend that patients with symptomatic first- to third-degree hemorrhoids initially be treated with increased fiber and adequate fluid intake and can be managed by various office procedures, including banding, sclerotherapy, and infrared coagulation and ligation [9].

Patients with fourth-degree or large third-degree hemorrhoids should be referred for hemorrhoidectomy surgery if they are refractory to or unable to tolerate office procedures [9].

Complications of untreated symptomatic hemorrhoid may include thrombosis, secondary infection, ulceration, abscess, and incontinence.

There is no published epidemiological study on hemorrhoidal diseases which categorized the risk factors in this region.

The aim of this retrospective study is to compile the demographic data in patients with hemorrhoid and assess the risk factors associated with this disease.

\section{Methods}

Place of study : Department of Surgery, J.K. Hospital associated with L.N. Medical College Bhopal (M.P.).

Type of study : Retrospective study

Sampling Method: Consecutive

Sample collection: Data were collected from medical record department. 430 adult patients with diagnosis of hemorrhoids, fulfilling all the inclusion criteria were included in this study. All the relevant details were obtained from medical record department with all demographic details.

Data were tabulated using detailed proforma. Following details of patients were recorded like age, sex, socioeconomic status, symptoms and risk factors. In all case records of admitted patients with hemorrhoids, final diagnosis was made on the basis of detailed history, clinical \& digital per rectal examination and proctoscopy.

External examination with inspection of the anal and perianal region was done to see external sentinel piles, prolapsed internal piles and fissure or perianal ulceration. On digital per rectal examination were usually done to rule out any pathology like rectal carcinoma, rectal polyp, hypertrophied anal papilla, thrombosed internal piles etc.

The anal sphincter tone was also assessed.Proctoscopy was usually done in each case to see the internal hemorrhoids with their location and to rule out any pathology. Conservative management was preferred in first, second and some third degree hemorrhoid.

Patients with fourth degree or large third-degree hemorrhoids were treated by open hemorrhoidectomy surgery or stapled hemorrhoidopexy. All the relevant collected data was compiled on master chart.

Inclusion criteria: Patients with hemorrhoid of age group 20 to 80 years who were admitted in surgery ward were included in the study

Exclusion Criteria: Patients with piles secondary to anorectal tumor, pregnant female patients and patients less than 20 years of age were excluded from study

Statistical Methods: Results were shown in tables, comparing their numbers and percentages by scientific calculator and standard appropriate statistical formula.

Ethical Permission: Yes 


\section{Results}

The aim of this study was to analyze statistics in patients with hemorrhoids to explain the demographic details and risk factors associated with hemorrhoids. Records of patients with hemorrhoids who were admitted in surgical wards were obtained from medical record department and after analysis the data following observations and results were obtained.

Table-1: Age wise distribution.

\begin{tabular}{|c|c|c|}
\hline Age group (years) & Total admission & \% \\
\hline $20-40$ & 198 & $46.0 \%$ \\
\hline $41-60$ & 136 & $31.6 \%$ \\
\hline $61-80$ & 96 & $22.3 \%$ \\
\hline
\end{tabular}

Age - Highest number of patients belongs to the young age group of 20-40 years.

Table-2: Sex Wise Distribution.

\begin{tabular}{|c|c|c|}
\hline & Total admission & \% \\
\hline Male & 297 & $69 \%$ \\
\hline Female & 133 & $31 \%$ \\
\hline & 430 & \\
\hline
\end{tabular}

Sex - The male to female ratio was $2.2: 1$. Out of total admission of $430,69 \%$ were male patients and $31 \%$ were female patients.

Table-3: Socioeconomic status wise distribution

\begin{tabular}{|c|c|c|}
\hline & Total admission & \% \\
\hline Lower & 142 & $33.0 \%$ \\
\hline Middle & 128 & $29.7 \%$ \\
\hline Upper & 160 & $37.2 \%$ \\
\hline Total & $\mathbf{4 3 0}$ & \\
\hline
\end{tabular}

Patients with higher socioeconomic status were most commonly affected group (37.2\%) with hemorrhoids.

Table-4: Symptoms.

\begin{tabular}{|l|c|c|}
\hline Complaints & Number of patients & \% \\
\hline$\bullet$ Bleeding per rectum & 290 & $67.5 \%$ \\
\hline$\bullet$ Pain during defecation & 230 & $53.5 \%$ \\
\hline$\bullet$ Pruritus ani & 160 & $37.2 \%$ \\
\hline$\bullet$ Discharge & 112 & $26.0 \%$ \\
\hline$\bullet$ Prolapsed swelling & 108 & $25.1 \%$ \\
\hline$\bullet$ Soiling & 40 & $9.3 \%$ \\
\hline
\end{tabular}

Most common symptoms were bleeding per rectum (67.5\%). Other symptoms were pain during defecation (53.5\%), pruritus ani (37.2\%), discharge (26.0\%), prolapsed swelling (25.1\%) and soiling $(9.3 \%)$ 
Original Research Article

Table-5: Common Risk Factors for haemorrhoids.

\begin{tabular}{|c|c|c|c|}
\hline & & Number of patients & $\%$ \\
\hline \multirow[t]{3}{*}{ Dietary habits } & Low fiber diet & 318 & $73.9 \%$ \\
\hline & Mixed diet & 289 & $67.2 \%$ \\
\hline & Poor hydration & 146 & $34 \%$ \\
\hline \multirow[t]{2}{*}{ Bowel habits } & Chronic constipation or diarrhea & 275 & $64 \%$ \\
\hline & Straining during defecation & 253 & $58.8 \%$ \\
\hline \multirow{2}{*}{$\begin{array}{l}\text { Amount of physical } \\
\text { activity }\end{array}$} & Low physical activity & 150 & $34.9 \%$ \\
\hline & Obesity & 177 & $41.1 \%$ \\
\hline
\end{tabular}

Risk factors commonly associated with hemorrhoidal disease include low fiber diet, chronic constipation, chronic diarrhea, chronic straining during defecation, pregnancy, sedentary lifestyle, habit of postponing the bowel movements, obesity and spinal cord injuries etc.

Table-6: Types of hemorrhoids present

\begin{tabular}{|l|l|c|c|}
\hline \multicolumn{2}{|c|}{} & Number of patients & $\%$ \\
\hline External hemorrhoid & 68 & $15.8 \%$ \\
\hline \multirow{3}{*}{ Internal hemorrhoid } & First degree & 11 & $2.5 \%$ \\
\cline { 2 - 4 } & Second degree & 55 & $12.7 \%$ \\
\cline { 2 - 4 } & Third degree & 150 & $34.8 \%$ \\
\cline { 2 - 4 } & Fourth degree & 146 & $33.9 \%$ \\
\hline
\end{tabular}

Diagnosis was based on external, digital per rectal examinations and proctoscopy; following findings were obtained.

Table-7: Management of haemorrhoids.

\begin{tabular}{|l|l|c|c|c|}
\hline \multirow{3}{*}{ Conservative management } & Procedure & & & \\
\cline { 1 - 4 } & Rubber band ligation & 18 & $4.1 \%$ & \multirow{2}{*}{$15.3 \%$} \\
\cline { 2 - 4 } & Sclerotherapy & 48 & $11.1 \%$ & \\
\hline \multirow{2}{*}{ Operative management } & Open hemorrhoidectomy & 350 & $81.4 \%$ & \multirow{2}{*}{$84.6 \%$} \\
\cline { 2 - 4 } & Stapled hemorrhoidopexy & 14 & $3.2 \%$ & \\
\hline
\end{tabular}

Management - Non-operative procedures were reserved for first degree, second-degree and early third degree hemorrhoids and are usually carried out on outpatient basis (15.3\%). Patients with fourth degree or large third-degree hemorrhoids were treated by open hemorrhoidectomy surgery or stapled hemorrhoidopexy (84.6\%).

\section{Discussion}

Hemorrhoids are common anorectal disease that affects about most of the population by the age of 50 years. Age wise distribution analysis of the data reveals that most common age group affected with hemorrhoids in our study was middle age group (41-60 years). It was almost similar to the study conducted by Ravindranath GG et al and Ali SA et al where most common age group was below 40 years $[10,11]$. This was in contrary with studies of Khan et al and Johanson et al [3, 12]. Sex wise distribution of hemorrhoids in our study reveals that the male to female ratio was $2.2: 1$. Out of total admission of $430,69 \%$ were male patients and $31 \%$ were female patients. This may be due to a greater likelihood of men seeking treatment for their hemorrhoids and embarrassment felt by women to consult for anorectal problems. A male predominance (66.7\%) compared to the females (33.3\%) suggested by Ravindranath GG et al [10]. A 55\% male predominance was also seen in a study by Ali SAet al [11]. Socioeconomic status had a significant effect on patients with hemorrhoids and our study showed that patients with higher socioeconomic status were most 


\section{Original Research Article}

commonly affected group (37.2\%) with hemorrhoids. According to Lohsiriwat $\mathrm{V}$ in a study, whites and higher socioeconomic status individuals were affected more frequently than blacks and those of lower socioeconomic status [13]. This association of haemorrhoid to higher socioeconomic status may be due to more prevalence of constipation due to dietary habits and lack of physical activity in this group. Also the patients with higher socioeconomic status usually have health seeking behavior.

The study of symptoms present in patients with hemorrhoids revealed that most of the patients had more than one complaint. The most common symptoms of hemorrhoids in the present study is bleeding per rectum (67.5\%) followed by pain during defecation (53.5\%), pruritus ani $(37.2 \%)$, discharge $(26 \%)$, prolapsed swelling (25.1\%) and soiling (9.3\%). Similar findings were suggested by Ali SA et al [11]. But this data was contradictory to Nikooiyan et al in a study where the most common symptoms was pruritus $(45.8 \%)$; other symptoms observed by him were discharge $(41.6 \%)$ and the anal pain $(22.5 \%)$ [2].

Risk factors evaluation in our study presents that the pathogenesis of hemorrhoids is not completely clear, but as stated by Kann et al, "all etiologic and risk factors work toward stretching and slippage of the hemorrhoidal tissue" [14]. As the supporting tissue of the anal cushions weakens, downward displacement of the cushions can occur, causing venous dilation and prolapse $[15,16]$. In the present study, risk factors for hemorrhoids were low fiber diet, mixed diet, poor hydration, chronic constipation or diarrhea, straining during the defecation, low physical activity and obesity.

Constipation and prolonged straining are the main risk factor to cause hemorrhoids in present study. Hard stool and increased intra-abdominal pressure could cause obstruction of venous return, resulting in engorgement of the hemorrhoidal plexus which leads to development of hemorrhoid [17].

Defecation of hard fecal material increases shearing force on the anal cushions. However, recent evidence questions the importance of constipation in the development of this common disorder [18]. Many investigators have failed to demonstrate any significant association between hemorrhoids and constipation, whereas some reports suggested that diarrhea is a risk factor for the development of hemorrhoids [12]. Low fiber intake, high intake of spicy and non-vegetarian mixed diet and poor hydration were found to be the risk factors of hemorrhoids in the present study. Increase dietary intake of fiber, vegetarian non spicy diet and increased hydration can improve and prevent the hemorrhoid by reducing the constipation which is a known risk factor for development of hemorrhoids. According to Anne F. Peery et al high grain fiber intake was associated with a reduced risk [19].

Sedentary life style, obesity and low physical activity was also one of the risk factors of hemorrhoids, which was corroborated by Khan et al in his study [3]. But according to Anne F. Peery et al, sedentary behavior was associated with a reduced risk, but not physical activity [19]. Neither being overweight nor obese was associated with the presence of haemorrhoids.

In our study, the analysis of types of hemorrhoids present was seen and diagnosis was made after doing examination of the patient. In present study, most of the admitted patients were related to external hemorrhoid, third and fourth degree internal hemorrhoids, which were operated. As most of the patients, with first and second degree were usually treated in outdoor with conservative treatment, so these cases were found to be less in the present study. In few cases they were admitted for conservative treatment and procedures like sclerotherapy and rubber band ligation in ward.

According to a study by Stefan Riss et al, out of the 976 participants, 380 patients (38.93\%) suffered from hemorrhoids. In 277 patients (72.89\%), hemorrhoids were classified as grade I, in 70 patients $(18.42 \%)$ as grade II, in 31 patients $(8.16 \%)$ as grade III, and in 2 patients $(0.53 \%)$ as grade IV [20].

There were two methods of management incorporated in our study based on the degree of hemorrhoid present. Two non-operative procedures were reserved for firstdegree, second-degree hemorrhoids and early third degree hemorrhoids, these cases were treated on outpatient basis and in some cases of admitted patients.

Sclerotherapy was most common non operative treatment, which were performed in 48 patients $(11.1 \%)$ by injecting chemical agents like $5 \%$ phenol in oil or hypertonic salt solution into the sub mucosa to induce the fibrosis [21]. Rubber band ligation was performed in selected patients $(4.1 \%)$ with third-degree hemorrhoids. Ligation of the hemorrhoidal tissue with a rubber band causes ischemic necrosis and scarring, leading to fixation of the connective tissue to the rectal wall. RBL can be safely performed in one or more than one place in a single session [22]. 


\section{Original Research Article}

Operative treatment were usually indicated when the conservative measures have failed with complication. Most common procedure performed in the present study was open hemorrhoidectomy (81.4\%). Excisional hemorrhoidectomy is the most effective treatment for hemorrhoids with the lowest rate of recurrence compared to other modalities [23].

Stapled hemorrhoidopexy was performed in only 14 patients $(3.2 \%)$ due to high cost of stapler. Stapled hemorrhoidopexy has been introduced since 1998 [24]. A circular stapling device is used to excise a ring of redundant rectal mucosa proximal to hemorrhoids and resuspend the hemorrhoids back within the anal canal.

A recent meta-analysis comparing surgical outcomes between stapled hemorrhoidopexyand hemorrhoidectomy, showed that stapled hemorrhoidopexywas associated with less pain, earlier return of bowel function, shorter hospital stay, earlier return to normal activities, and better wound healing, as well as higher degree of patient satisfaction[25].

\section{Conclusion}

Symptomatic Hemorrhoids are one of the common benign diseases usually found in patients with risk factors like chronic constipation, improper dietary habits, lack of physical activity and obesity. So the patients should be educated to change his dietary habits, to increase his daily physical activity and to prevent the constipation.

\section{Contribution from authors}

- Dr Vikas Kumar Malviya formulated the aims \& objectives with study design.

- Dr Tarun Kumar Sainia contributed to the preparation of the manuscript and Data analysis.

- Dr Shivam Diwan helped in data collection from medical record department.

- Dr Ashwin Apte supervised and guided for study

What this study add to existing knowledge: This demographic study of hemorrhoidal disease in this region can guide us to better understanding of the trends of this disease, which is most commonly encountered in our society. This useful information may aid in the assessment and definitive care of these patients with haemorrhoids.

Conflict of interest: None declared.

Funding: Nil, Permission from IRB: Yes

\section{References}

1. Marx JA, Hockberger RS, Walls R. Rosen's Emergency Medicine: Concepts and Clinical Practice. 6th ed. Philadelphia, Pa: Elsevier; 2006. 1509-12.

2. Nikooiyan P, Sardo HM, Poursaeidi B, Zaherara M, Ahmadi B. Evaluating the safety, efficacy and complications of electrotherapy and its comparison with conventional method of hemorrhoidectomy. Gastroenterology and Hepatology from bed to bench. 2016; 9 (4):259-67.

3. Khan RM, Itrat M, Ansari AH, Zulkifle M, Ethisham. A study on associated risk factors of haemorrhoids. J Biol Sci Opinion. 2015;3(1):36-8.

4. Banov Jr L, Knoepp Jr LF, Erdman LH, Alia RT. Management of hemorrhoidal disease. Journal of the South Carolina Medical Association (1975). 1985 Jul; 81 (7): 398.

5. Jacobs D. Clinical practice. Hemorrhoids. N Engl J Med. 2014 Sep 4;371(10):944-51.doi:10.1056/ NEJMcp 1204188 .

6. Rivadeneira DE, Steele SR, Ternent C, et al. Practice parameters for the management of hemorrhoids (revised 2010). Dis Colon Rectum. 2011 Sep;54(9):1059-64. doi: 10.1097/DCR.0b013e318225513d.

7. Altomare DF, Roveran A, Pecorella G, et al. The treatment of hemorrhoids: guidelines of the Italian Society of Colorectal Surgery. Tech Coloproctol. 2006 Oct; 10 (3):181-6. Epub 2006 Sep 20. DOI:10.1007/s 10151 - 006-0277-y

8. Buntzen S, Christensen P, Khalid A, et al. Diagnosis and treatment of haemorrhoids. Dan Med J. 2013 Dec; 60 (12): B4754.

9. Wald A, Bharucha AE, Cosman BC, ACG clinical guideline: management of benign anorectal disorders. Am J Gastroenterol. 2014 Aug; 109 (8): 1141-57; (Quiz) 1058. doi: 10. 1038/ajg. 2014. 190. Epub 2014 Jul 15.

10. Wald A, Bharucha AE, Cosman BC, et al. ACG clinical guideline: management of benign anorectal disorders. Am J Gastroenterol. 2014 Aug;109 (8): 114157; (Quiz) 1058. doi: 10. 1038/ajg. 2014.190. Epub 2014 Jul 15. 


\section{Original Research Article}

11. Ali SA, Shoeb MF. Study of risk factors and clinical features of hemorrhoids. International Surgery Journal. 2017 May 24;4(6):1936-9.

12. Johanson JF, Sonnenberg A. Constipation is not a risk factor for hemorrhoids: a case-control study of potential etiological agents. Am J Gastroenterol. 1994 Nov; 89(11):1981-6.

13. Lohsiriwat V. Hemorrhoids: from basic pathophysiologyto clinical management. World J Gastroenterol. 2012 May 7; 18 (17):2009-17. doi: 10. 3748/ wjg.v18.i17.2009.

14. Kann BR, Whitlow CB. Hemorrhoids: diagnosis and management.Techniques in Gastrointestinal Endoscopy. 2004 Jan 1; 6 (1):6-11.
15. Lestar B, Penninckx F, Kerremans R. The composition of anal basal pressure. An in vivo and in vitro study in man.Int J Colorectal Dis.1989;4(2):118-22

16. Loder PB, Kamm MA, Nicholls RJ, et al. Haemorrhoids: pathology, pathophysiology and aetiology. Br J Surg. 1994 Jul;81(7):946-54.

17. Loder PB, Kamm MA, Nicholls RJ, et al. Haemorrhoids: pathology, pathophysiology and aetiology. Br J Surg. 1994 Jul; 81(7):946-54.

18. Johanson JF, Sonnenberg A. The prevalence of hemorrhoids and chronic constipation. An epidemiologic study. Gastroenterology. 1990 Feb; 98 (2): $380-6$

\section{How to cite this article?}

Malviya V.K, Diwan S, Sainia T.K, Apte A. Demographic study of hemorrhoid with analysis of risk factors. Surgical Update: Int J surg Orthopedics.2019;5(1):7-13.doi:10.17511/ijoso.2019.i01.02. 